\title{
Sports-related traumatic brain injuries: mini review
}

\begin{abstract}
Traumatic brain injuries are common with sports-related injuries. The signs and symptoms vary from mild to severe with examples of a mild headache or nausea alone to more severe symptoms such as coma or death. Furthermore, studies have shown chronic repercussions of traumatic brain injuries such as Alzheimer's disease or dementia late in life. Immediate evaluation and a timely diagnosis is the most important in the treatment of sports- related brain injuries since it has been proven to lead to a better prognosis following the injury. Promoting health awareness with an emphasis on education, injury prevention, early recognition, and effective management of injuries may avert premature death and devastating early onset of neurodegenerative disease.
\end{abstract}

Keywords: alzheimer's disease, traumatic brain injuries, concussion, sports-related brain injuries, dementia, helmets
Volume 4 Issue 3 - 2016

\author{
Nye Mary Katherine, ' Wagilla Osman,' Kara \\ Ellison,2 Mack Leigh J, ${ }^{3}$ Brown Tony ${ }^{4}$ \\ International Clinical Research Organization, USA \\ ${ }^{2}$ Advanced Research and Human Development, USA \\ ${ }^{3}$ American Biotech Labs, USA \\ ${ }^{4}$ Columbia University, USA
}

Correspondence: Tony Brown, Columbia University, USA,

Email tbrown@post.harvard.edu

Received: December 31, 2015 | Published: February 18, 2016
Abbreviations: MBTI, mild traumatic brain injury; TBI, traumatic brain injuries; ICCS, international conference on concussion sport; LOC, loss of consciousness

\section{Introduction}

A traumatic brain injury is one of the major acquired brain ailments with devastating effects on all segments of the population. Whereas young adults acquire the vast majority of brain injuries through participation in contact associated sports, falling is the leading cause in the senescent community. ${ }^{1}$ Nonetheless, the effect of even minor brain injury may result in everlasting life altering effects.

In recent decades, much attention has been given to sport related brain injuries. Minor brain injury involving contact sports became the concerning theme of discussions within major social media outlets. It has been suggested that traumatic brain injury may lead to early onset of brain disorders including dementia and Alzheimer disease. ${ }^{2}$ Countless former athletes of contact sports have suggested that they, too, are suffering from premature cognitive deterioration. The healthcare community has also recognized traumatic brain injury as a major cause of morbidity and mortality in young adults. Increased frequency of hospitalization in conjunction with an escalation in rates of morbidity and mortality, especially within the geriatric population, is igniting public concern. Implementing meaningful methods of prevention, early recognition of clinical manifestation, timely application of necessary treatment and intervention can all lead to better prognoses of functional restoration and averting death. Granted significant evolution has been made in implementing protocols and guidelines to achieve better prevention, detection, and treatment, more research is needed to identify key elements. Identified biomarkers are better used to determine potential risk factors which, in turn, may be able to predict symptom onset and progression of associated neurodegenerative disorders.

\section{Discussion}

Traumatic brain injury is one of the major current public health concerns. In recent decades, ongoing discussion has persevered in the medical community and society as a whole. Issues channeling though major journals of medicine and a plethora of TBI related themes of discussion linger throughout social media. According to Phillips \& Woesssner, 1.7 million treated traumatic brain injuries are documented annually with an estimated 3 million being left either unrecognized or untreated, of which 173,285 are derived from sports or recreational- related traumatic brain injury. ${ }^{3}$ Sports related traumatic brain injuries have garnered more attention in social media and medical literature in recent years. This may difficult be due in part to an increased body of scientific literature and growing concern for the long-term effects of the injuries.

The Center for Disease Control and Prevention are supplementing the term, "concussion" with the term mild traumatic brain injury (MBTI). The International Conference on Concussion Sport (ICCS) 2012 census statement describes a concussion as "a complex pathophysiological process affecting the brain, induced by biomechanical forces". ${ }^{4}$ The ICCS also described a concussion involving neuropathological changes, and emphasized that the acute clinical symptoms reflect a functional disturbance rather than a structural injury. Concussions are believed to account for $80 \%$ of traumatic brain injuries (TBI) visits to emergency departments. In 2013, the American Medical Society for Sports Medicine reported up to 3.8 million sports-related concussions per year with up to $50 \%$ going unreported. ${ }^{5}$ The findings of a study conducted to identify the severity and frequency of TBI occurring in high school sports:

"Of 23,566 reported injuries in the 10 sports during the 3 -year study period, 1219 (5.5\%) were MTBIs, football accounted for 773 (63.4\%) of cases; wrestling, 128 (10.5\%); girls' soccer, $76(6.2 \%)$; boys' soccer, 69 (5.7\%); girls' basketball, 63 (5.2\%); boys' basketball, 51 (4.2\%); softball, 25 (2.1\%); baseball, 15 (1.2\%); field hockey, 13 (1.1\%); and volleyball, $6(0.5 \%)$. The injury Rates per 100 player -seasons were 3.66 for football, 1.58 for wrestling, 1.14 for girls' soccer, 1.04 for girls' basketball, 0.92 for boys' soccer, 0.75 for boys' basketball, 0.46 for softball, 0.46 for field hockey, 0.23 for baseball, and 0.14 for volleyball. The median time lost from participant for all MTBIs was 3 days. There were 6 cases of subdural hematoma and intracranial injury reported in football. Based on these data, an estimated 62,816 cases of MTBI occur annually among high school varsity athletes participating in these sports, with football accounting for about $63 \%$ of cases". ${ }^{6}$

There are various types of traumatic brain injuries (TBI). Severity can range from mild to severe, dependent upon mechanism or force of injury and anatomical factors. One may not experience any significant loss of consciousness, only a mild headache, with or without nausea and demonstrate a full recollection of the incident. More severe cases can involve loss of consciousness (LOC) or amnesia as well as abnormalities in movement and behavior. The onset of maladies may be obvious within the first few moments of an athlete being 
examined, while others can be insidious and not manifest for days, weeks, or months. Numerous professional athletes have reported negative neurological outcomes years after departing from the sport. Although presentation can vary greatly, most sources will agree onset is characterized as sudden.

Dependent upon the mechanism of injury and prior physical conditions, the specific impairment may vary. TBI can result from a chemical insult, hypoxia, infection, or tumor. However, more often, in terms of athletics, a person may suffer a closed head injury due to a sudden deceleration or acceleration and less likely from a penetration injury. Furthermore, recurrent injuries can have a cumulative effect. There have been many studies performed revealing the late life consequences of TBI. For example, it is thought that repeated TBI affects the aging of the brain. The association of TBI with an increased risk of Alzheimer's disease and other neurodegenerative disorders years after the injuries has been reported with epidemiologic studies. ${ }^{7}$ These studies have shown the risk of dementia progressively increases as the number and severity of head injury increased.

As one would expect, in a closed head injury, there is no penetration. The protective, bone or cranium remains intact. Damage is not focal but more diffuse. An example of a mechanical injury would be a sudden start -to-stop or stop-to-start. Any action that causes the brain to strike or forcibly move against the inside of the skull has the potential to create damage. Axons may be stretched beyond their physical capacity and actually pull to the point of tearing, causing irreparable damage. Due to the disruption of neurofilament structures impair axonal swelling, Wallerian degeneration, and transection. A release of excitatory neurotransmitters acetylcholine, glutamate, and aspartate as well as free radicals have been found to contribute to secondary injuries. ${ }^{9}$ Concussions are the best case scenario of a TBI. By definition, it is a mild form of brain injury. The dysfunction is temporary and the likelihood that the person will return to prior functioning capacity is great. The effects are usually short lasting without life- long impact. LOC in such cases is $<10 \%$. The injured may complain of a headache or seem dazed, sometimes unable to recall circumstances prior to or immediately following the accident. Symptoms may be apparent up to 2 weeks.

Other signs and symptoms of a MTBI include a headache as well as nausea. Observers may notice slurred speech or a difference in personality. Again, this has a great opportunity to resolve in time. In more extreme cases, the athlete might seize or go into a coma due to the loss of consciousness. If the small vessels are torn, blood will start to collect and form a hematoma or blood clot. Depending upon the location, further neurological signs (such as posturing), cognitive impairment, or the loss of thinking as well as emotional liability. Effects can linger, become permanent and in some cases progress.

Athletes could suffer a cerebral contusion or a "bruising" to the brain caused by a direct blow to the head, causing the brain to bounce against the inside of the skull and bruise the brain tissue. ${ }^{10}$ MTBIs are functional and not a structural injury which cannot be detected through diagnostic imaging; therefore, computed tomography (CT) and magnetic resonance imaging (MRI) will be normal. ${ }^{11}$ However, imaging is still warranted in all cases with severe mechanism, significant loss of consciousness, focal neurologic deficit, or worsening symptoms. ${ }^{10} \mathrm{CT}$ scans are the quickest acute diagnostic method to detect cerebral contusions because it can be performed immediately in the hospital, usually complete in less than 5 minutes, and has fairly exact findings. ${ }^{12}$ MRIs are more sensitive and accurate compared to $\mathrm{CT}$ scans due to the multiplanar capabilities and greater sensitivities for edema; however, some facilities do not contain an MRI machine and cannot be performed on an emergent basis. MRI findings have been found to typically identify the lesions from the onset of the injury and are sensitive to hyperacute hemorrhagic contusions, which occur within 12 hours of the injury.

While various imaging techniques, such as the CT and MRI, can evaluate the anatomy of the brain, other modalities must be employed to assess function. An EEG or electroencephalogram is used to measure and record the electrical activity in the brain. Depending upon the speed of the rhythm in a given location, one may deduce an abnormality such as an area effected by trauma. While rhythms can vary slightly from each person, there is a general pattern when an individual is awake, active, at rest, or sleeping. In one study, it was concluded a shift in the spectral profile with higher frequencies in the frontal brain regions when compared with a healthy individual. ${ }^{13}$ When exploring the possibility of injury, there needs to be a means of comparison. A recording technique called quantitative electroencephalogram, QEEG, will measure one subject with those in the same population. By analyzing the variances, damage or injuries might be confirmed. There are other confounding causes that can cause an abnormal study, therefore the standard use of an EEG is not in practice at this time consistently. Evoked potentials and event related potentials can also be examined to aid in diagnosis since it has been concluded to demonstrate abnormal brain functions with TBI. ${ }^{14}$ Electrophysiological abnormalities are noted with the EEG; however, additional research is needed to further identify the EEG findings and TBI impairments.

Outcomes vary from minor ailments to more severe life-long injuries or even death. Short term effects include a mild headache, nausea, or feeling light headed for a few days. Whereas long term injuries can be more serious and require surgical interventions, rehabilitation, and close monitoring. Approximately 52,000 of the 1.7 million American with head injuries die. ${ }^{15}$ If the TBI resulted in a coma, recovery will be longer and rehabilitation more extensive. In a majority of cases, the greater length of time the person remains comatose, the greater probability of death and permanent neurological damage. The prognosis of patient with a TBI depends on the mechanism of the injury and the initial treatment. Therefore it is more important to prevent TBI from occurring.

Due to the various acute and chronic sequela associated with TBI, there have been many recommended prevention methods and within each sport many changes continue to occur to prevent cerebral contusions and traumatic brain injuries. The individual athlete still remains their own, best prevention against injury. One example of prevention of sports related TBI is the use of sport- specific helmets during the game. The use of helmets has been found to reduce head injuries in sports such as baseball, ice hockey, rugby, alpine skiing, and snowboarding. ${ }^{16-19}$ In contrast, soccer players wearing helmets did not reduce the rate of head injuries. ${ }^{20}$ Traumatic brain injuries in all sports has lead to extensive design improvements to the players' helmets to prevent or lessen the severity of the injuries, and reduced the risk of cerebral contusions. Examples of improved helmet design changes included an increase in the padding around the skull or a chin strap which keeps the helmet more snug. In some instances, the use of protective equipment adversely affected playing behavior and encouraged more risky behavior so the risk of injury actually increased. ${ }^{21,22}$ Football players also wear helmets; however, there are no specific helmet modifications, which have been identified to reduce concussions. ${ }^{23}$ Motorcycle and bicycle helmets have shown to reduce the severity of accident related head injuries. National sports are also making changing to rules in order to protect their players from sports related TBI. For example the NFL has made changes to rules and 
increased repercussions for blows to the head of a defenseless receiver or helmet to helmet contact. ${ }^{24}$

\section{Conclusion}

Traumatic brain injury remains one of our major public health concerns. The significant or minor brain injury warrants a great deal of attention. Failure of timely recognition or delay implementation of necessary treatment may lead to negative consequences which have the potential of lasting throughout a lifetime. The astute assessment of signs and symptoms of traumatic brain injury needed for early recognition and accurate diagnosis, may lead to significant reduction of late development of TBI associated neurodegenerative disorders including Alzheimer disease, prevent major devastating disabilities or avert premature death. The development and application of advanced concussion assessment tools including neuropsychological testing, neuroimaging, balance and gait assessment; there is a rising amount of data pertaining to the prevention of TBIs and special emphasis in clinical practices and management of concussion patients. Research suggests close monitoring and immediate medical evaluation lead to a better lifelong prognosis. Recurrent brain injuries may lead to more acute life threatening injuries, increase risk TBI associated late life altering sequelae, including a post-concussion syndrome, headaches, epilepsy, and vertigo. Timely recognized and appropriately treated sports related brain injuries can have an excellent prognosis. Promoting public awareness with emphasis on education and injury prevention, early recognition and effectively manage of injuries, may avert the premature death and devastating early onset of neurodegenerative disorders.

\section{Acknowledgments}

None.

\section{Conflicts of interest}

None.

\section{Funding}

None.

\section{References}

1. Thompson HJ, McCormick WC, Kagan SH. Traumatic Brain Injury in Older Adults: Epidemiology, Outcomes, and Future Implications. J Am Geriatr Soc. 2006;54(10):1590-1595.

2. Dams-O'Connor K1, Cuthbert JP, Whyte J, et al. Traumatic Brain Injury among Older Adults at Level I and II Trauma Centers. J Neurotrauma. 2013;30(24):2001-2013.

3. Phillips S, Woessner D. Sports-relatede Traumatic Brain Injury. Prim Care. 2015;42(2):243-248.

4. McCrory P, Meeuwisse WH, Aubry M, et al. Consensus statement on concussion in sport: the 4th International Conference on Concussion in Sport held in Zurich, November 2012. Br J Sports Med. 2013;47(5):250-258.
5. Clark M, Guskiewicz K. Sport-Related Traumatic Brain Injury. In: Laskowitz D \& Grant G (Eds.), Translational Research in Traumatic Brain Injury: Frontiers in Neuroscience. CRC Press/Taylor and Francis Group, Boca Raton, Florida, USA. 2016.

6. Harmon KG, Drezner JA, Gammons M, et al. American Medical Society for Sports Medicine position statement: concussion in sport. BR J Sports Med. 2013;47(1):15-26.

7. Powell JW, Barber-Foss KD. Traumatic Brain Injury in High School Athletes. JAMA. 1999; 282(10):958-963.

8. Plassman BL, Grafman J. Traumatic brain injury and late-life dementia. Handb Clin Neurol. 2015; 128:711-722.

9. Povlishock JT, Katz DI. Update of neuropathology and neurological recovery after traumatic brain injury. J Head Trauma Rehabil. 2005;20(1):76-94.

10. Hayes RL, Dixon CE. Neurochemical changes in mild head injury. Semin Neurol. 1994;14(1):25 -31.

11. McCrea HJ, Perrine K, Niogi S, et al. Concussion in Sports. Sports Health . 2013;5(2):160-164.

12. Guskiewicz KM, Broglio SP. Acute sports-related traumatic brain injury and repetitive concussion. Handb Clin Neurol. 2015;127:157-172.

13. Moralews DL. Brain Contusion Imaging. MedScape. 2015.

14. Balkan O, Virji-Babul N, Miyakoshi M, et al. Source-domain spectral EEG analysis of sports-related concussion via Measure Projection Analysis. Conf Proc IEEE Eng Med Biol Soc. 2015; 2015:4053-4056.

15. Arciniegas DB, Anderson CA, Topkoff J, et al. Mild traumatic brain injury: a neuropsychiatric approach to diagnosis, evaluation, and treatment. Neuropsychiatr Dis Treat. 2005;1(4):311-327.

16. http://www.mdguidelines.com/cerebral-contusion-closed

17. Johnston KM, McCrory P, Mohtadi NG, et al. Evidence-Based review of sport-related concussion: clinical science. Clin J Sport Med. 2001;11(3):150-159.

18. Sulheim S, Holme I, Ekeland A, et al. Helmet use and risk of head injuries in alpine skiers and snowboarders. JAMA. 2006;295(8):919-924.

19. Russell K, Christie J, Hagel BE. The effect of helmets on the risk of head and neck injuries among skiers and snowboarders: a meta-analysis. CMAJ. 2010;182(4):333-340.

20. Hollis SJ, Stevenson MR, McIntosh AS, et al. Incidence, risk, and protective factors of mild traumatic brain injury in a cohort of Australian nonprofessional male rugby players. Am J Sports Med. 2009;37(12):2328-2333

21. McIntosh AS, McCrory P. Impact energy attenuation performance of football headgear. Br J Sports Med. 2000;34(5):337-341.

22. Finch CF, McIntosh AS, McCrory P. What do under 15 year old schoolboy rugby union players think about protective headgear? $\mathrm{Br} J$ Sports Med. 2001;35(2):89-94.

23. Hagel B, Meeuwisse W. Risk compensation: a "side effect" of sport injury prevention? Clin J Sport Med. 2004;14(4):193-196.

24. http://www.nytimes.com/2010/10/21/sports/football/21helmets. html?pagewanted $=$ all 